\title{
Principal components' features of mid-latitude geomagnetic daily variation
}

\author{
P. De Michelis ${ }^{1,2}$, R. Tozzi ${ }^{1}$, and G. Consolini ${ }^{3}$ \\ ${ }^{1}$ Istituto Nazionale di Geofisica e Vulcanologia, 00143 Roma, Italy \\ ${ }^{2}$ Dip. Scienze della Terra, Università degli Studi di Siena, 53100 Siena, Italy \\ ${ }^{3}$ INAF-Istituto di Fisica dello Spazio Interplanetario, 00133 Roma, Italy
}

Received: 10 June 2010 - Revised: 6 December 2010 - Accepted: 9 December 2010 - Published: 21 December 2010

\begin{abstract}
The ionospheric and magnetospheric current systems are responsible of the daily magnetic field changes. Recently, the Natural Orthogonal Components (NOC) technique has been applied to model the physical system responsible of the daily variation of the geomagnetic field, efficiently and accurately (Xu and Kamide, 2004). Indeed, this approach guarantees that the number of parameters used to represent the physical process is small as much as possible, and consequently process control for such system becomes apparent.

We focus our present study on the analysis of the hourly means of the magnetic elements $\mathrm{H}, \mathrm{D}$ and $\mathrm{Z}$ recorded at L'Aquila observatory in Italy from 1993 to 2004 . We apply to this dataset the NOC technique to reconstruct the 3dimensional structures of the different ionospheric and magnetospheric current systems which contribute to the geomagnetic daily variations. To support our interpretation in terms of the different ionospheric and magnetospheric current systems, the spectral and statistical features of the timedependent amplitudes associated to the set of natural orthogonal components are analyzed and compared to those of a set of descriptors of the magnetospheric dynamics and solar wind changes.
\end{abstract}

Keywords. Geomagnetism and paleomagnetism (Time variations, diurnal to secular) - Magnetospheric physics (Current systems) - General or miscellaneous (Techniques applicable in three or more fields)

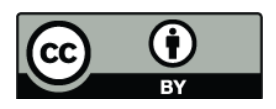

Correspondence to: P. De Michelis (paola.demichelis@ingv.it)

\section{Introduction}

The geomagnetic field varies on a huge range of time scales: from milliseconds to millions of years. The slower changes, occurring over time scales of a few years to thousand years, are related to the dynamo processes acting within the Earth and are generally referred to as geomagnetic secular variation. On the contrary, the short-term variations are primarily of external origin arising from currents flowing in the ionosphere and magnetosphere (Merrill et al., 1996). Currents flowing in the magnetosphere are responsible for the occurrence of geomagnetic storms and substorms (i.e. of irregular variations), while currents flowing in the ionosphere are associated with a more or less regular daily variation of the geomagnetic field.

Among the possible short-term geomagnetic variations the smoothest and most regular is that observed on magnetically quiet days, and it is known as "solar quiet daily variation". This variation mainly arises from the ionospheric current system flowing in the so-called dynamo region. This current system, which can be quite well approximated by a 2-D current flowing in the ionospheric E-region between 90 and $130 \mathrm{~km}$ (Chapman, 1929; Richmond et al., 1976), is driven by different processes. This current is indeed related with the expansion and contraction of the atmosphere as the Sun rises and falls daily through the year, with the global scale horizontal upper-atmosphere winds, with the lunar tidal forces upon the region, and with variations of the sun electromagnetic emissions responsible for extra fotoionization of the region. As a result, the solar daily variation is a function of latitude, local time, season and solar activity level (Campbell, 2003).

However, on days characterized by normal geomagnetic activity, or even days with only minor disturbance, in addition to the solar quiet daily variation there is the solar disturbance variation, whose intensity varies with the intensity

Published by Copernicus Publications on behalf of the European Geosciences Union. 
of the general disturbance. The solar daily variation fluctuates in both amplitude and pattern even on very quiet days. These changes are known as "day-to-day variation" and are ascribed to irregularities in the winds at E-region height and to solar-activity-related changes in the ionospheric conductivity and wind system. Moreover, ground magnetometers are capable of detecting fields due to distant magnetospheric sources such as the magnetotail current, the partial ring current, the substorm current wedge and the dayside current wedge. The contribution coming from these magnetospheric sources is not entirely negligible. Indeed, they physically contribute to the daily variation even on relatively quiet days.

At present, the accurate determination of quiet-day field variation finds utility in improving the satellite main-field modelling, in profiling the Earth's electrical conductivity, and in determining the baselines from which magnetospheric disturbances are quantified. Moreover, monitoring the dayto-day variability could provide very important contributions to the knowledge of the ionospheric dynamics as it could be the key to investigate the solar wind-magnetosphereionosphere coupling from a different point of view.

Recently, Xu and Kamide (2004) and Chen et al. (2007) have used the method of Natural Orthogonal Components (NOC) to decompose the magnetic daily variation and express it as the summation of eigenmodes or Empirical Orthogonal Functions (EOFs). In Xu and Kamide (2004) the NOC analysis is applied to the horizontal magnetic field component $(\mathrm{H})$ recorded at Beijing Ming Tombs observatory (BMT), while in Chen et al. (2007) the H-component from a meridian chain of magnetometers along $120^{\circ} \mathrm{E}$ longitude is analyzed. In both papers the first NOC eigenmode represents the solar quiet daily variation, the second the disturbancedaily variation, while the third and fourth eigenmodes may be related to specific currents in the magnetosphere.

Driven by the above results, we focus our present study on the analysis of the hourly means of the three magnetic field elements (H, D and Z) recorded at L'Aquila observatory over a period of 12 years. The aim of our study is not only to identify the different current systems that contribute to the daily variation of the Earth's magnetic field, but also to study their mid/long-term temporal evolution and find possible correlations with appropriate parameters related to these currents, by analyzing the spectral and statistical features of the associated PCs. Last but not least, we investigate the crosstalk among the different principal components, obtained via the NOC decomposition, by estimating an information theory quantity: the mutual information.

\section{Dataset and analysis}

To investigate the daily variation of the geomagnetic field and its temporal evolution, we analyze the hourly means of the magnetic elements $\mathrm{H}, \mathrm{D}$ and $\mathrm{Z}$ recorded in Italy at L'Aquila (AQU) observatory from 1993 to 2004. The position of the geomagnetic observatory (corrected geomagnetic coordinates at the epoch 2007: GLat $36.24^{\circ} \mathrm{N}$ and GLong 87.2 ${ }^{\circ} \mathrm{E}$ ) is particularly suitable for this type of study being sufficiently far away from the polar regions, where the magnetospheric processes may completely dominate the geomagnetic field recordings and, only on rare occasions, the true solar daily variation may be observed.

To reveal simple patterns within the complex geomagnetic daily variation we apply the NOC method (Jackson et al., 1991; Golovkov et al., 1992; Xu and Kamide, 2004). This method offers the way to extract those structures that remain coherent throughout a time series. In practice, a set of orthogonal eigenvectors and eigenvalues is estimated from observed data. By conveniently combining these eigenvectors it is possible to write the observed variables in terms of the smallest possible set of natural orthogonal basis functions. This type of analysis has been widely used in literature, for instance for the study of daily magnetic variation (Golovkov et al., 1978; Xu and Kamide, 2004), for space-time analysis of the main geomagnetic field (Rotanova et al., 1982), for the study of global models of the geomagnetic field (Xu, 2002, 2003), for the automatic calculation of $\mathrm{K}$ indices (Golovkov et al., 1989; Papitashvili et al., 1992), and even for the separation of the substorm current system into directly driven and loading-unloading components (Sun et al., 1998, 2000). Similar techniques have been applied to the study of the solar cycle (Mininni et al., 2002, 2004; Consolini et al., 2009).

The starting point of the NOC technique is the assumption that we measure a variable $x\left(d_{i}, t_{j}\right)$ representing a magnetic field element $\left(\mathrm{H}, \mathrm{D}\right.$ or Z) on a certain day $d_{i}$ at the time $t_{j}$ (here being the local time - LT). Given a number of samples of $x\left(d_{i}, t_{j}\right)$, NOC allows us to define a smaller set of variables/functions, named Empirical Orthogonal Functions (EOFs) and Principal Components (PCs), capable of describing the entire set of observations. Actually, there are many methods capable of doing this job, the benefit of NOC is that the set of functions used in the expansion of the time series is not determined in advance but, conversely, is estimated using the dataset. Therefore, the daily variation of any magnetic element can be written in terms of a basis of Empirical Orthogonal Functions (EOFs) $\phi^{k}\left(t_{j}\right)$ as follows:

$x\left(d_{i}, t_{j}\right)=\sum_{k=1}^{N} A^{k}\left(d_{i}\right) \phi^{k}\left(t_{j}\right)$

where the collection of values $x\left(d_{i}, t_{j}\right)$ provides the elements $x_{i j}$ of the $m \times n$ matrix $\mathbf{X}$ with rows corresponding to the observations made on a fixed day $d_{i}$ and at $n$ values of time $t_{j}$, and the columns to the observations made at fixed time $t_{j}$ and on $m$ different days $d_{i}$, and $N$ is the number of components chosen for the decomposition (i.e., the truncation level). In Eq. (1), the EOF is $\phi^{k}\left(t_{j}\right)$ that is the mode of the $k$-th component with elements $\phi_{j}^{k}(j=1,2, \ldots, n)$ describing the temporal distribution (i.e., it is the basis used for the expansion), and the PC is $A^{k}\left(d_{i}\right)$, which is the amplitude of the 
corresponding mode with elements $a_{i}^{k}$ for $(i=1,2, \ldots, m)$. In practice, the EOFs $\phi^{k}\left(t_{j}\right)$ and the PCs $A^{k}\left(d_{i}\right)$ (in the following $A_{i}^{k}$ and $\phi_{j}^{k}$, respectively) are capable of identifying the components of the daily variation and their temporal evolutions.

To evaluate the EOFs $\phi^{k}$ and the associated PCs $\boldsymbol{A}^{k}$ from a dataset, it is necessary to minimize the error made in the representation of observed data by means of the expansion of Eq. (1). This error $\delta$ can be defined as the total squared difference between observed and estimated data (see $\mathrm{Xu}$ and Kamide, 2004), and it is given by:

$\delta=\sum_{i=1}^{m} \sum_{j=1}^{n}\left[x_{i j}-\sum_{k=1}^{N} A_{i}^{k} \phi_{j}^{k}\right]^{2}$.

The NOC approach consisting of minimizing $\delta$ by solving the following eigenvector/eigenvalue problem,

$\mathbf{V} \boldsymbol{\phi}^{k}=\lambda_{k} \boldsymbol{\phi}^{k}$

where $\mathbf{V}$ is the $N \times N$ covariance matrix with elements $v_{i j}$ given by

$\mathbf{V}=\mathbf{X}^{\mathrm{T}} \mathbf{X}$

where $\mathbf{X}=\left\{x_{i j}\right\}$, and $\mathbf{X}^{\mathrm{T}}$ is the transpose matrix.

Thus, solving Eq. (3) we estimate the eigenvalues $\lambda^{k}$ and the corresponding eigenvectors $\boldsymbol{\phi}^{k}$ for $k=1, \ldots, N$, and successively the amplitudes $A^{k}$, given by

$\boldsymbol{A}^{k}=\mathbf{X} \boldsymbol{\phi}^{k}$.

where $\boldsymbol{A}^{k}=\left\{A_{j}^{k}\right\}$.

We note that the eigenvalues $\lambda_{k}$ provide a measure of the variance of the corresponding PC, i.e., $\lambda_{k}=N\left\langle y_{k}^{2}\right\rangle$, where $\left\langle y_{k}^{2}\right\rangle$ is the mean-square value of the $k$-th PC. Furthermore, once the eigenvalue spectrum $\lambda_{k}$ is evaluated, it is possible to establish the number $N^{*}<N$ of fundamental EOFs that are sufficient to capture most of the properties of the observed variable, i.e.,

$x\left(d_{i}, t_{j}\right) \sim \sum_{k=1}^{N^{*}} A^{k}\left(d_{i}\right) \phi^{k}\left(t_{j}\right)$.

On the basis of the above consideration, it is clear that the NOC analysis preserves the total variance (energy) of the signal, and that it is strictly valid for signals caused by the linear superposition of orthogonal modes. Consequently, the results of its application to signals resulting from nonlinear processes could be questionable, and the interpretation of the meaning of the EOFs may be difficult.

\section{Results and discussion}

\subsection{NOC results}

Figure 1 shows the hourly means of $\mathrm{H}, \mathrm{D}$ and $\mathrm{Z}$ magnetic field elements recorded at L'Aquila geomagnetic observatory

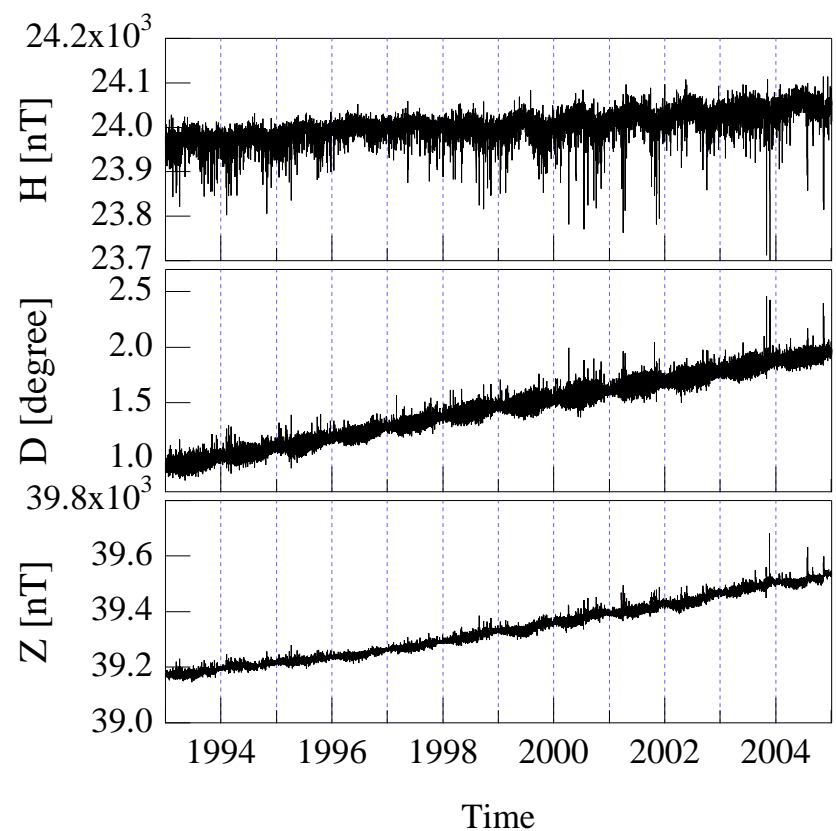

Fig. 1. Hourly means of H, D and $\mathrm{Z}$ magnetic field elements (from top to bottom) as recorded at L'Aquila observatory in the period from January 1993 to December 2004.

from 1993 to 2004. The shown trends evidence the existence of annual changes (see modulation in amplitude) as well as of secular changes (the average trend). Before applying the NOC analysis we eliminated these trends removing the daily mean from each day.

Figure 2 shows the spectra of the eigenvalues $\lambda_{k}$ evaluated from Eq. (3) for the H, D and Z magnetic field elements, respectively. All the spectra are characterized by a rapid drop at small $k(k<5)$. Indeed, the first 4 eigenvalues (and equivalently the associated first $4 \mathrm{PCs}$ ) explain up to $75 \%, 88 \%$ and $90 \%$ of the total variance of the $\mathrm{H}, \mathrm{D}$ and $\mathrm{Z}$ magnetic field elements, respectively. Consequently, we can assume that most of the variability observed in the geomagnetic field daily variation is explained in terms of a very small number of natural orthogonal components $(k<5)$. The rest of the eigenvalues can either take into account transient fluctuations and noise or represent the contribution of stochastic processes. This point is confirmed by the exponential decay of the eigenvalue spectra $\left[\lambda_{k} \sim \exp (-\alpha k)\right]$ for $k>10$. We notice that the spectrum of the eigenvalues $\lambda_{k}$ associated with the horizontal component $\mathrm{H}$ is different from the others. In the eigenvalue spectrum of the $\mathrm{H}$ component the first 2 eigenvalues are of the same order of magnitude. In contrast, in the case of the $\mathrm{D}$ and $\mathrm{Z}$ elements eigenvalue spectra the largest eigenvalue is one order of magnitude larger than the second one. Thus, in this last case, the daily variation is essentially represented by the first EOF. Considering the rapid drop of the eigenvalue spectra, we fix the truncation level $N^{*}=4$ for all the three magnetic field elements being 


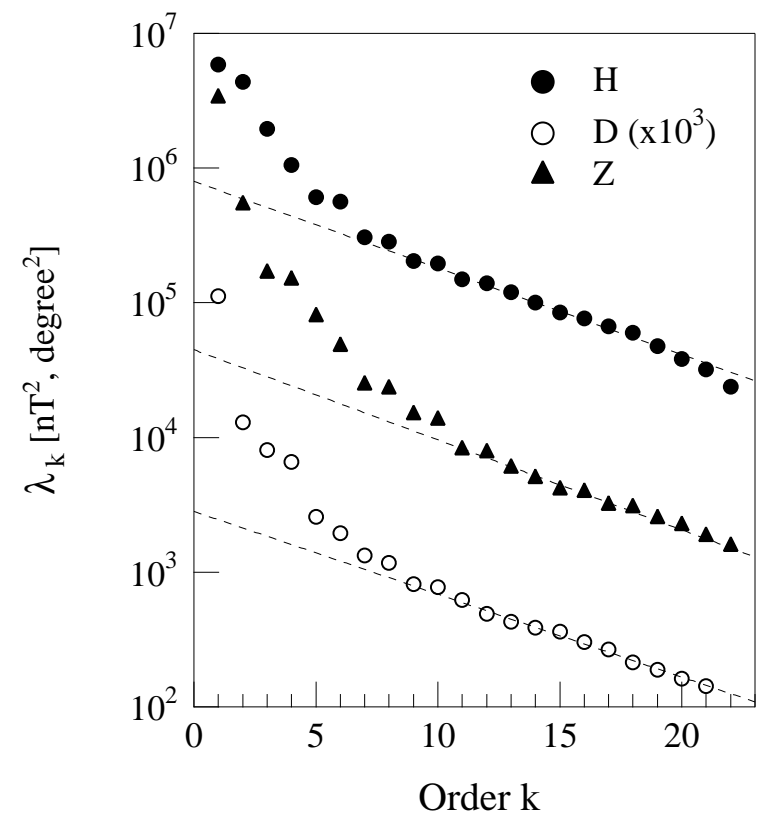

Fig. 2. The NOC eigenvalue spectra $\lambda_{k}$ of $\mathrm{H}, \mathrm{D}$ and $\mathrm{Z}$ magnetic field elements. The $\lambda_{k}$ spectra of declination $\mathrm{D}$ has been scaled by a factor $10^{3}$ for convenience. The dashed lines are exponential fits of spectra in the range $k \in[10,21]$ characterized by a characteristic decay factor $\alpha=[0.148 \pm 0.006]$.

the first four EOFs capable of representing the major part of the daily variation.

In the following figures, we group the EOFs according to the different current systems we think they may represent. Thus, in Fig. 3 we report the first EOF associated with D $\left(\phi_{\mathrm{D}}^{1}(t)\right)$ and $\mathrm{Z}\left(\phi_{\mathrm{Z}}^{1}(t)\right)$ elements and the second EOF associated with the $\mathrm{H}\left(\phi_{\mathrm{H}}^{2}(t)\right)$ component. These EOFs describe the solar daily variation along the three magnetic field elements. The trend of these EOFs is that of the solar quiet daily variation $\left(S_{\mathrm{q}}\right)$ expected at mid-latitude where the AQU geomagnetic observatory is located (Campbell, 2003). Indeed, the daily pattern of the $\phi_{\mathrm{H}}^{2}(t)$ function is characterized by a morning minimum (at about 09:00 LT) and an afternoon maximum (at about 20:00 LT). In contrast, the pattern of the $\phi_{\mathrm{D}}^{1}(t)$ function exhibits a morning maximum (at about 08:00 LT) and an afternoon minimum (at about 14:00 LT), while the $\phi_{Z}^{1}(t)$ function is characterized by a minimum at around noon.

The PCs $\left(A_{\mathrm{H}}^{2}, A_{\mathrm{D}}^{1}\right.$ and $\left.A_{\mathrm{Z}}^{1}\right)$, associated with the EOFs reported in Fig. 3, are plotted in Fig. 4. These PCs (or daily amplitudes) exhibit the well-known feature of seasonal solar daily variation characterized by a maximum in the springsummer period and a minimum in the autumn-winter one (Matsushita and Maeda, 1965). The seasonal variation can be better visualized using the statistical method of superposed epoch analysis. The results of this method, applied to the daily values of the amplitudes, point out the characteristics of the signal on 1-year scale as shown in the right panels of

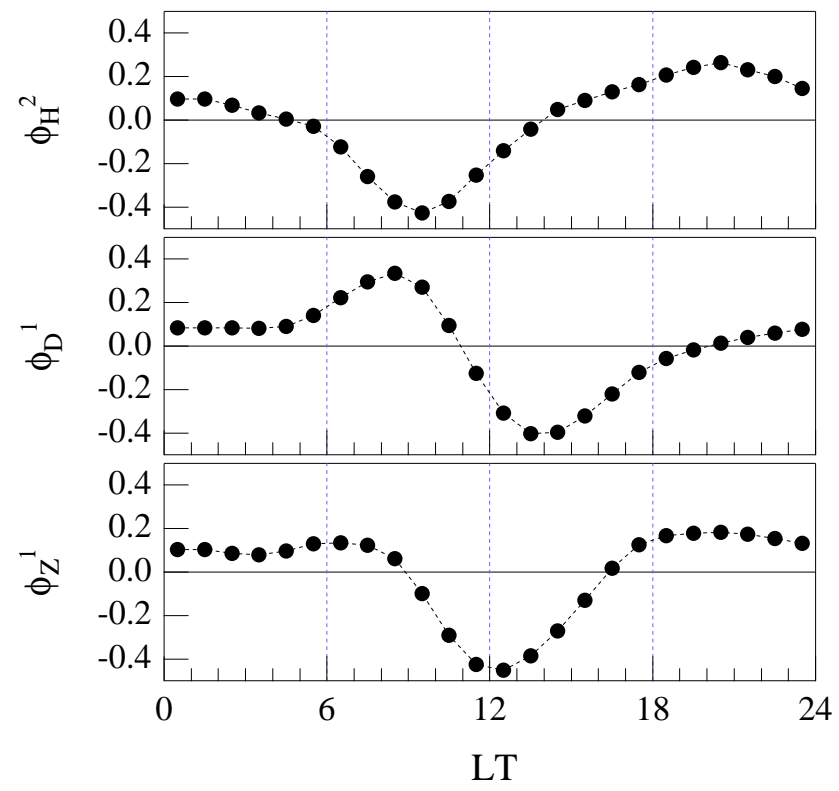

Fig. 3. The EOFs associated with the solar quiet daily variation for $\mathrm{H}, \mathrm{D}$ and $\mathrm{Z}$ magnetic field elements.

Fig. 4. The observed seasonal asymmetry is the same characterizing the solar daily variation and it is particularly evident in the $\mathrm{H}$ and $\mathrm{Z}$ components. In the past few decades, it was implicitly assumed that the daily variation was a function of the Sun's declination (Howe, 1950), so that spring and autumn equinoxes were symmetrical from a geomagnetic point of view. Nowadays, it is known that the seasonal dependence of the daily variation is more complicated. Recently, Chulliat et al. (2005) have found a strong lack of symmetry about the summer solstice for the geomagnetic field diurnal variation at mid-latitude observatories. In particular, the average seasonal variations of the 24-h line amplitude for the $\mathrm{H}$ and $\mathrm{Z}$ components (the only investigated by the authors) relative to Chambon-La-Forét observatory is well in agreement with our results. It has been suggested (Chulliat et al., 2005) that the seasonal asymmetry could correspond to a seasonal asymmetry in the lower thermospheric winds responsible for the solar daily variations through the ionospheric dynamo.

It is known that the $S_{\mathrm{q}}$ field varies slowly in amplitude and phase (time of maximum) through the months of the year. Naturally, by means of NOC we obtained a trend of the $S_{\mathrm{q}}$ (as represented by the EOFs) that is a mean trend over the investigated time interval along each of the three considered magnetic field elements. While NOC decomposition is able to reconstruct the seasonal variations of $S_{\mathrm{q}}$, it is not capable of reproducing its slow variation in phase. This can be verified in Fig. 5 where, to support our results, we have reported both the true and the NOC-reconstructed $S_{\mathrm{q}}$ for all the magnetic elements. The reconstructed $S_{\mathrm{q}}$ has been obtained multiplying each EOF for the corresponding PC amplitude $\left(S_{\mathrm{q}}^{j} \sim A_{j}^{k} \phi_{j}^{k}\right.$ with $\left.j=H, D, Z\right)$ for the year 1996 , 

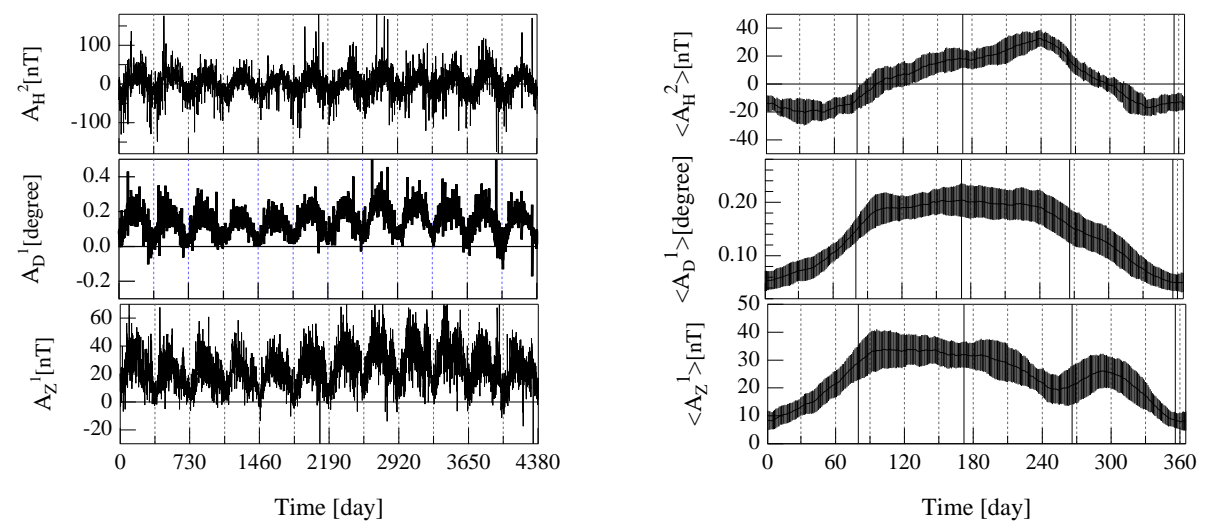

Fig. 4. The PCs associated with the EOFs reported in Fig. 3 for H, D and Z magnetic field elements (left panels) and the average annual behavior of PCs as evaluated applying the superposed epoch analysis (right panels). The vertical solid lines in right panels indicate the equinoxes and solstices.

which corresponds to a solar minimum. With this operation we are practically including the seasonal variation. The true $S_{\mathrm{q}}$, estimated for each magnetic field element, is nothing else than the average daily variation of $S_{\mathrm{q}}$ reconstructed using $\mathrm{H}$, $\mathrm{D}$ and $\mathrm{Z}$ values recorded at L'Aquila geomagnetic observatory during the five quietest days of each month of 1996. In detail, we have identified the five quietest days of each month of the 1996 year and averaged over them. In this way, the $S_{\mathrm{q}}$ variation reconstructed using NOC analysis can be properly compared with the $S_{\mathrm{q}}$ variation estimated directly from observatory data. The comparison confirms the possibility of the NOC analysis to reconstruct the mean trend of $S_{\mathrm{q}}$ field with its seasonal variation. However, some discrepancies can be observed as, for instance, those regarding the shift of $S_{\mathrm{q}}$ phase. In the second part (Sects. 3.2 and 3.3) of this paper it will be indicated that the effect of the ionospheric current system responsible of the $S_{\mathrm{q}}$ field may probably influence different EOFs. We suggest that the small contributions, which we can find in the EOFs of higher order, could take into account of the slow phase variations.

The EOFs $\phi_{\mathrm{H}}^{1}(t), \phi_{\mathrm{D}}^{2}(t)$ and $\phi_{\mathrm{Z}}^{2}(t)$ are reported in Fig. 6. These EOFs represent the disturbance daily variation SD as defined by Chapman and Bartels (1940). According to the authors (Chapman and Bartels, 1940), this variation is related to a current system present in the ionosphere and magnetosphere that corresponds to the partial ring current and the related field-aligned currents. Indeed, the EOFs $\phi_{\mathrm{H}}^{1}(t)$ and $\phi_{\mathrm{Z}}^{2}(t)$ are characterized mostly by a variation in the dusk and midnight sectors that is related to the partial ring current flowing in the equatorial plane, while the EOF $\phi_{\mathrm{D}}^{2}(t)$ is asymmetric with respect to the local midnight showing a maximum in the evening sector (at about 21:00 LT) and a minimum in the early morning one (at about 07:00 LT). The different sign of the function reflects the opposite senses of the field-aligned currents. Indeed, the field-aligned currents go down to the ionosphere on the dawn side, and go up to the magnetotail on the dusk side, flowing in the opposite way at the latitude where AQU geomagnetic observatory is located.

The intensity of these currents depends on the magnetic activity level. Consequently, we expect that this dependence characterizes also the PCs $\left(A_{\mathrm{H}}^{1}, A_{\mathrm{D}}^{2}\right.$ and $\left.A_{\mathrm{Z}}^{2}\right)$ associated with these components.

As in the previous case, we apply the method of the superposed epoch analysis to the PCs for the whole period (19932004). The results indicate a seasonal trend (see Fig. 6) that may be described as a double wave with a minimum in MayJune and two maxima localized around February-March and September-October, respectively. The annual trend of this variation is similar to that own of Dst-index (data not shown). This magnetic activity index, which is computed from measurements of the $\mathrm{H}$ component at four low-latitude ground stations widely spaced in longitude, is designed to measure the azimuthally symmetric part of the low-latitude perturbation field due to magnetic disturbances (Sugiura and Kamei, 1991). It includes the disturbance field produced by the ring current although it is affected also by the disturbance fields generated by the magnetopause current, the cross-tail current, the field-aligned currents and the currents induced in the diamagnetic Earth (Campbell, 1996). Dst-index is independent of ionospheric conductivity and instead of one or two peaks per year at the solstices, it is characterized by two peaks near the equinoxes. The similarity between the annual variation of magnetic activity, described by the Dstindex, and the average annual variation of the PCs supports our interpretation on the physical processes associated with the EOFs in terms of SD variation.

Thus, to completely identify the Empirical Orthogonal Functions we looked at both the daily trends of these functions and the annual trends of the associated Principal Components. Indeed, latitudinal and temporal variations of the $S_{\mathrm{q}}$ and $S_{\mathrm{D}}$ fields are very different from each other and consequently are easily identifiable. For this reason we think 

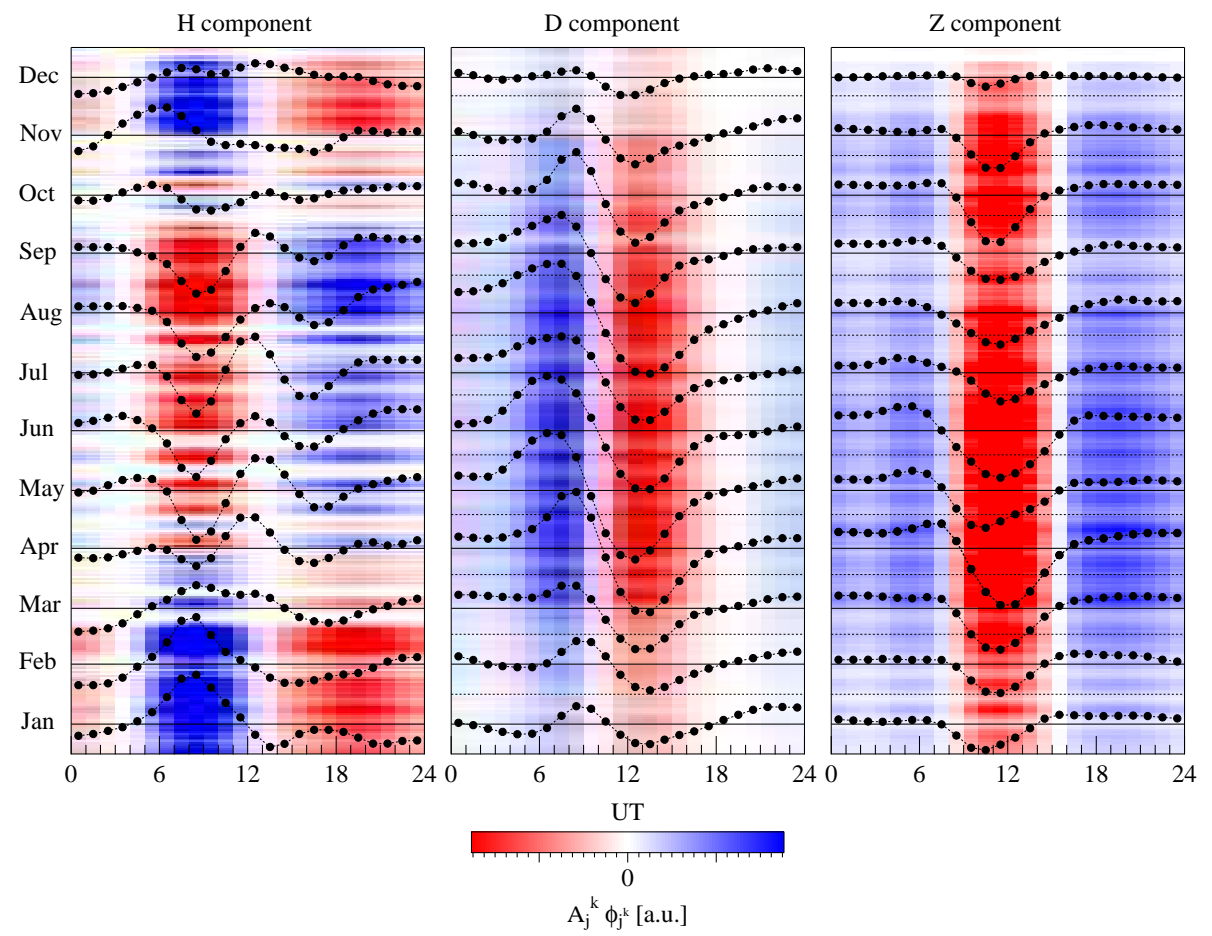

Fig. 5. Comparison between the seasonal dependence of the 1996 quiet daily variation of the three magnetic field elements (H, D and Z) as reconstructed by multiplying each EOF for the corresponding amplitude PC (negative and positive values are in red and blue, respectively) for the year 1996 and the true $S_{\mathrm{q}}$ (black circles) evaluated by averaging the daily variation of the 5 quietest days of every month. Solid horizontal lines refers to the average $S_{\mathrm{q}}$ values for the true daily variation. A moving average over 5 days has been applied on the $A_{j}^{k}$ to reduce the noise for visualization purposes. Time is reported in universal time $(\mathrm{UT}=\mathrm{LT}-1)$ for convenience.

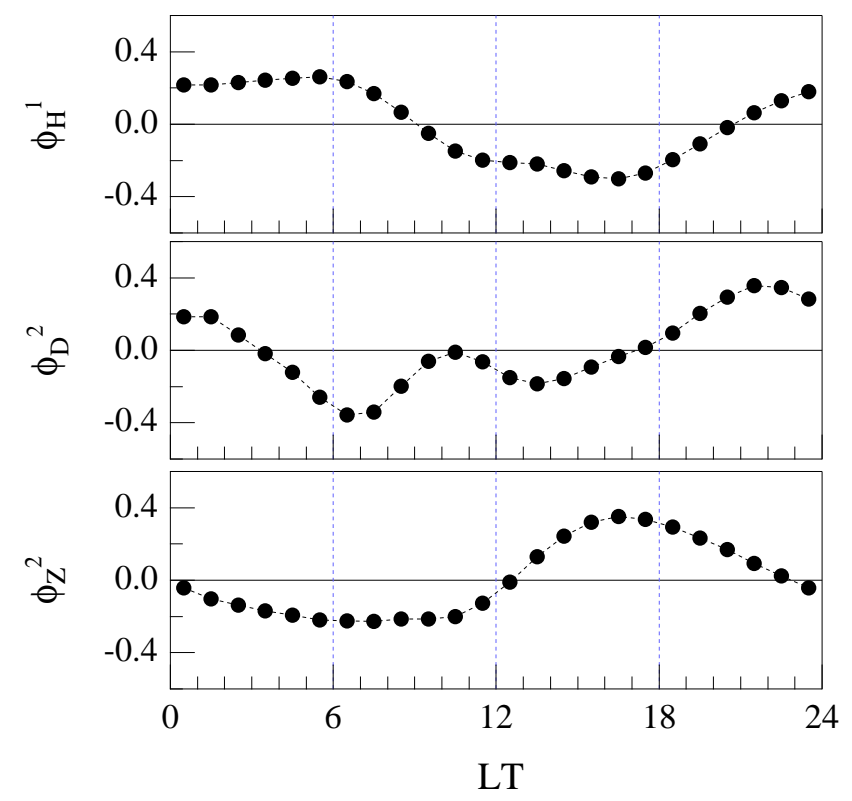

Fig. 6. The EOFs associated with the disturbance daily variations for $\mathrm{H}, \mathrm{D}$ and $\mathrm{Z}$ magnetic field elements. that our interpretation of the first and second EOF should be correct although Xu and Kamide (2004) obtained a different result from the NOC decomposition of the horizontal $\mathrm{H}$ component. Indeed, in Xu and Kamide (2004) the first and second NOC eigenmodes and associated PCs for the $\mathrm{H}$ component correspond to $S_{\mathrm{q}}$ and $S_{\mathrm{D}}$, respectively. The observed difference can be due to the following reasons: (i) a different latitudinal location for the observations (there is a difference of $7^{\circ}$ in latitude); (ii) a different time interval for the considered dataset (1 year in Xu and Kamide, 2004, 12 years in our case). Furthermore, we notice that in our case the first two terms of the eigenvalue spectrum are of the same order of magnitude meaning that the energies associated with these terms are comparable and making the exchange of these two terms not relevant.

The EOFs $\phi_{\mathrm{H}}^{3}(t), \phi_{\mathrm{D}}^{3}(t)$ and $\phi_{\mathrm{Z}}^{3}(t)$ (Fig. 8) are characterized by significant variations on the dayside $(6 \div 18 \mathrm{LT}) \mathrm{im}$ plying their relationship with the dayside currents. Considering the different current systems present in the dayside of the magnetosphere, the only current capable of producing such a daily variation is the magnetopause current system. This current system, located at the Earth's magnetopause, forms the physical boundary between the solar wind plasma and the magnetosphere plasma. It can be visualized as closed loops of current flowing around two null or neutral points, where 

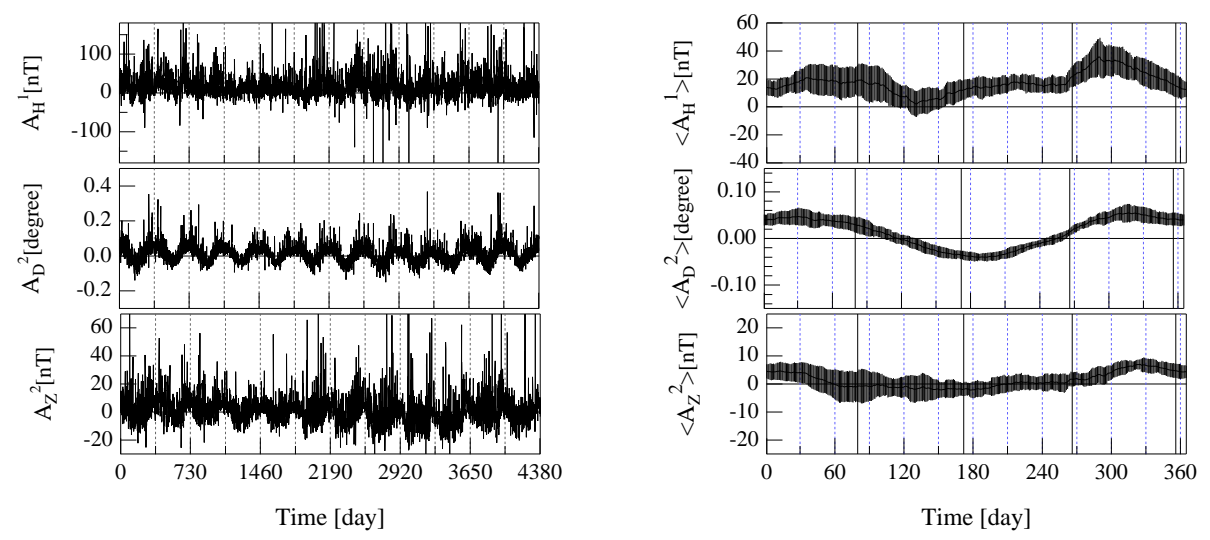

Fig. 7. The PCs associated with the EOFs reported in Fig. 6 for the 3 magnetic field elements (left panels) and the average annual behavior of PCs as evaluated applying the superposed epoch analysis (right panels). The vertical solid lines in right panels indicate the equinoxes and solstices.

the total magnetic field (main field plus the field from the magnetopause currents) is zero. The currents flow clockwise in the Southern Hemisphere and anticlockwise in the Northern one. The effect of the Earth's rotation under this current system gives rise to a geomagnetic daily variation which depends on both the intensity of the current and the angle between the Earth's dipole axis and the direction of the solar wind. The seasonal variation of the magnetopause current system (see Fig. 9) is clearly visible in the trend of the associated PCs $\left(A_{\mathrm{H}}^{3}, A_{\mathrm{D}}^{3}\right.$ and $\left.A_{\mathrm{Z}}^{3}\right)$. It is characterized by two maxima near the equinoxes (see the plot relative to the $\left\langle A_{Z}^{3}\right\rangle$ ), thus supporting the independence of the associated EOFs from the ionospheric processes, which are mainly characterized by a single maxima structure localized in the summer season.

Finally, Figs. 10 and 11 show the EOFs and the associated PCs obtained for $K=4$, respectively. We are not capable of offering a definite physical interpretation for these EOFs on the basis of the NOC analysis alone. It is reasonable to state that these functions represent the combined effects of different magnetospheric current systems that the NOC method is not able to single out.

\subsection{Relation of PCs with solar, interplanetary and magnetospheric parameters}

To complete our interpretation of EOFs in terms of the various ionospheric and magnetospheric current systems, we investigate the relation between daily variation of the EOFs (i.e., PCs) and some solar, interplanetary and magnetospheric parameters that influence or describe the evolution of these current systems. To investigate such a relation (shared information), we consider the daily averages of the following quantities

- the interplanetary magnetic field Z-component,

- the Y-component of the electric field $E_{\mathrm{y}}^{\mathrm{SW}}$,

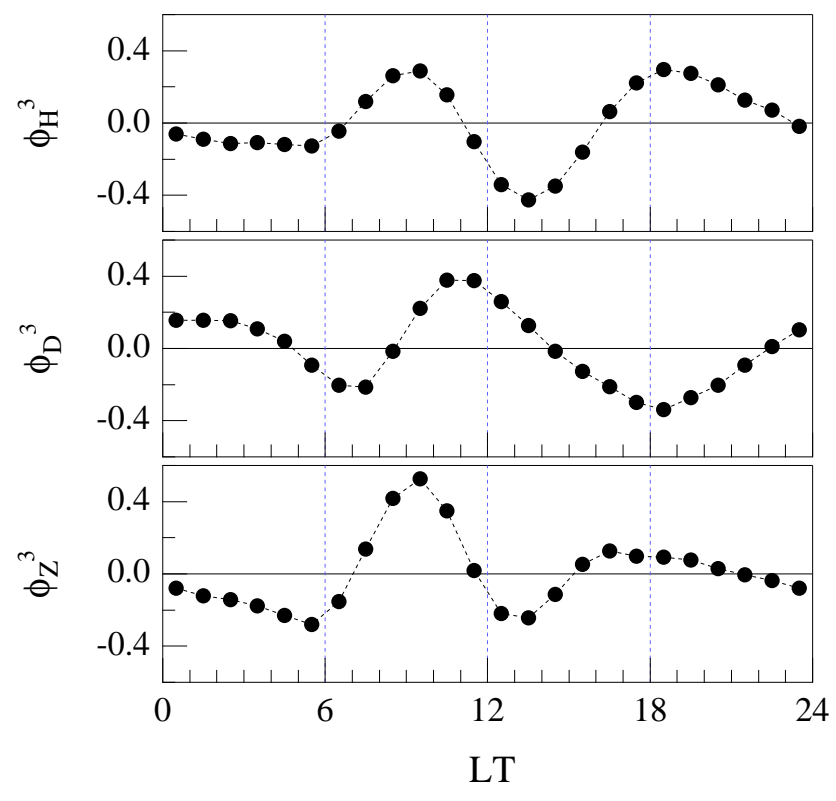

Fig. 8. The EOFs associated with the contribution to the daily variation due to the magnetopause current system and relative to $\mathrm{H}, \mathrm{D}$ and $\mathrm{Z}$ magnetic field elements.

- the Dst-index,

- the dynamical pressure of the solar wind $P_{n}^{\mathrm{SW}}$,

- the F10.7 solar flux density, measured at a wavelength of $10.7 \mathrm{~cm}$,

for the period 1993-2004. Data come from the NSSDC/OMNI database.

The standard way to evaluate the mutual interrelation between two signals is to compute their linear cross-correlation coefficient (the Pearson's coefficient $|r|$ ). As well known, this coefficient $|r|$ provides a simple measure of the linear correlation and it ranges from 0 to 1 . A value of 1 is 

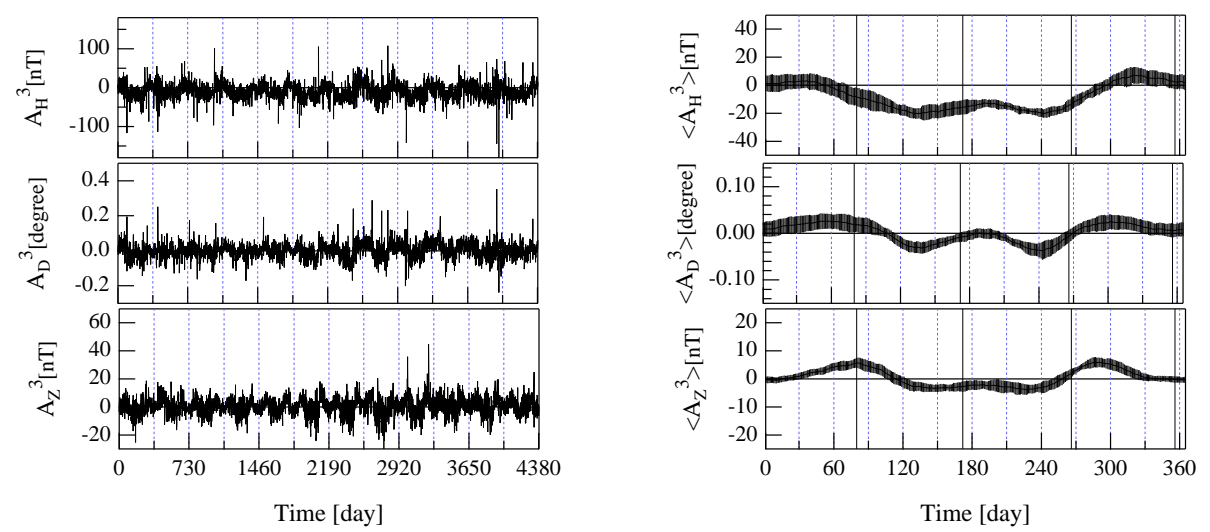

Fig. 9. The PCs associated with the EOFs reported in Fig. 8 for H, D and Z magnetic field elements (left panels) and the average annual behavior of PCs as evaluated applying the superposed epoch analysis (right panels). The vertical solid lines in right panels indicate the equinoxes and solstices.

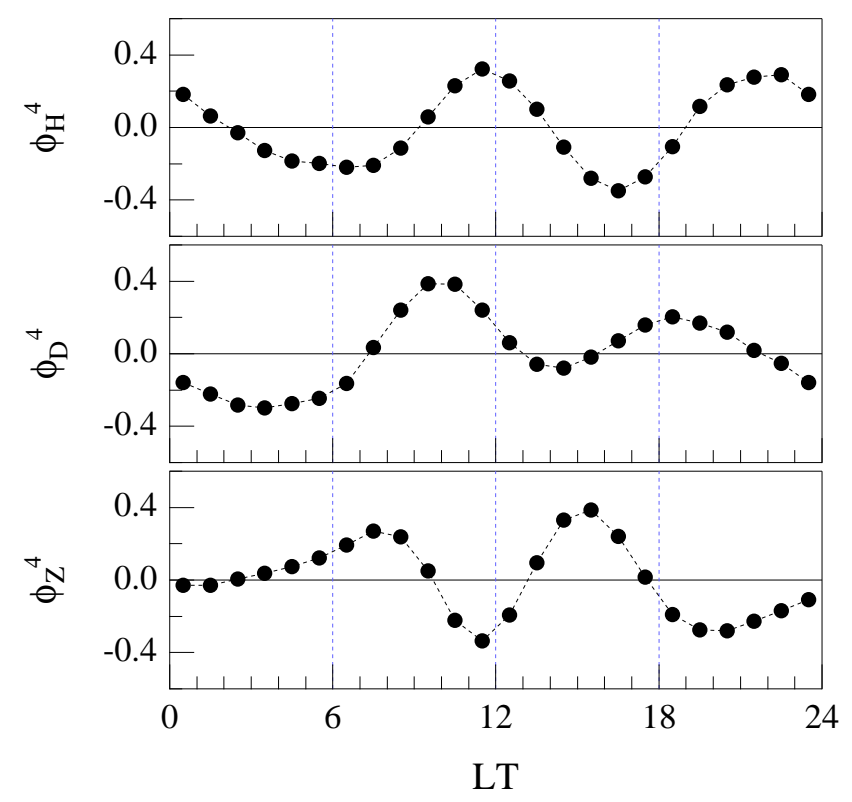

Fig. 10. The EOFs associated with the contribution to the daily variation due to other magnetospheric current systems and relative to $\mathrm{H}, \mathrm{D}$ and $\mathrm{Z}$ magnetic field elements.

the result of a perfect linear relationship between the analyzed variables, while a value of 0 is the result of no linear relationship. In practice, the value of this parameter is some intermediate number whose significance depends on the number of samples. We recall that in statistics a result is called statistically significant if it is unlikely to have occurred by chance. Therefore, it is crucial to establish if the value of the Pearson's coefficient between two signals is statistically significant. So, once established the null hypothesis as $|r|=0$, i.e. absence of correlation, and chosen a significance level, what we here call the significance threshold (or p-value) must be estimated (of course for the selected significance level and for the degrees of freedom of the problem, usually $N-2$, where $N$ is the sample size). This means that if the found value of correlation coefficient is less than the specified threshold the hypothesis of correlation must be rejected. Thus, in order to establish a significance level for the correlation found, we have estimated a significance threshold $\left|r_{\mathrm{s}}\right|$ for the Pearson's coefficient by means of the wellknown Surrogate Data Test (SDT) (Theiler et al., 1992). This significance threshold corresponds to the standard 5\% nullhypothesis value for two uncorrelated noise samples. In detail, for each couple of variables we first generate a couple of random phase signals with the same Fourier spectral density, by simply randomizing the Fourier phases of the original signals. Successively, we estimate the Pearson's coefficient $|r|$. We iterate this procedure for 1000 times generating a set of 1000 Pearson's coefficient values for each couple of variables, over which we evaluate the 5\% significance level. That means that in our case correlation values smaller than the corresponding 5\% SDT significance value can be read as absence of correlation. In Table 1 we report only the statistically significant correlation coefficients between the PCs and the selected parameters. Looking at the values reported in Table 1 it could be conjectured that they are not representative of a relevant correlation degree. However, although the significance must be evaluated in respect of the null-hypothesis threshold, as already explained above, there is also a physical reason for the observed low values of $|r|$. As a matter of fact, as well documented in a huge literature the response of the magnetosphere-ionosphere system to the changes of the external (boundary) conditions is nonlinear. In this case, the Pearson's coefficient $|r|$, which is designed to estimate the presence of a linear correlation, could not be capable of capturing the overall (linear and nonlinear) correlation degree. For this reason we approach the evaluation of the correlation degree via the null-hypothesis test.

According to the results reported in Table 1 we note that the first group of PCs $\left(A_{\mathrm{H}}^{2}, A_{\mathrm{D}}^{1}\right.$ and $\left.A_{\mathrm{Z}}^{1}\right)$ is correlated with 

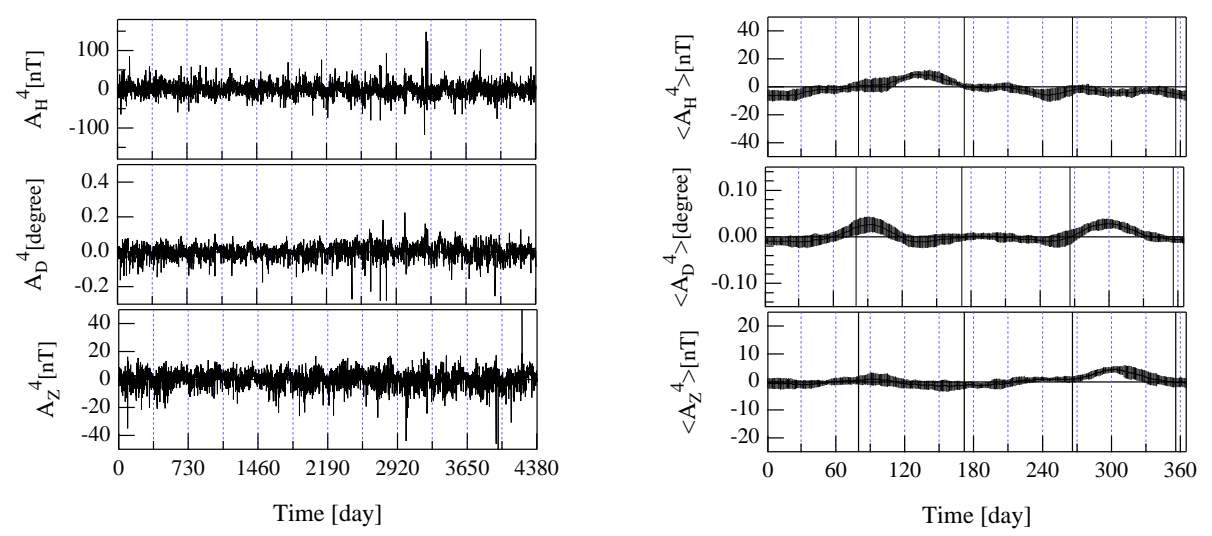

Fig. 11. The PCs associated with the EOFs reported in Fig. 10 for H, D and Z magnetic field elements (left panels) and the average annual behavior of PCs as evaluated applying the superposed epoch analysis (right panels). The vertical solid lines in right panels indicate the equinoxes and solstices.

Table 1. The Pearson's correlation coefficient $|r|$. The "_" refers to values less than the fixed 5\% null-hypothesis threshold value. Bold values identify correlation values higher than 3 times the 5\% threshold value.

\begin{tabular}{cccccc}
\hline $\mathrm{PC}$ & $\mathrm{F} 10.7$ & $B_{\mathrm{Z}}^{\mathrm{SW}}$ & $E_{\mathrm{y}}^{\mathrm{SW}}$ & $P_{n}^{\mathrm{SW}}$ & Dst \\
\hline$A_{\mathrm{H}}^{2}$ & 0.047 & - & - & 0.176 & - \\
$A_{\mathrm{D}}^{1}$ & $\mathbf{0 . 3 0 2}$ & 0.054 & - & - & - \\
$A_{\mathrm{Z}}^{1}$ & $\mathbf{0 . 3 2 8}$ & - & - & - & - \\
& & & & & \\
$A_{\mathrm{H}}^{1}$ & - & $\mathbf{0 . 2 7 6}$ & $\mathbf{0 . 3 6 9}$ & $\mathbf{0 . 2 0 3}$ & $\mathbf{0 . 4 8 7}$ \\
$A_{\mathrm{D}}^{2}$ & 0.098 & $\mathbf{0 . 2 2 2}$ & $\mathbf{0 . 2 6 5}$ & $\mathbf{0 . 2 2 3}$ & $\mathbf{0 . 4 9 5}$ \\
$A_{\mathrm{Z}}^{2}$ & $\mathbf{0 . 1 4 3}$ & 0.082 & $\mathbf{0 . 1 7 6}$ & $\mathbf{0 . 2 5 6}$ & $\mathbf{0 . 2 8 2}$ \\
$A_{\mathrm{H}}^{3}$ & - & 0.081 & 0.067 & - & - \\
$A_{\mathrm{D}}^{3}$ & $\mathbf{0 . 1 5 9}$ & 0.054 & 0.084 & 0.139 & - \\
$A_{\mathrm{Z}}^{3}$ & 0.062 & - & - & - & 0.177 \\
$A_{\mathrm{H}}^{4}$ & 0.100 & - & - & 0.051 & - \\
$A_{\mathrm{D}}^{4}$ & $\mathbf{0 . 1 4 2}$ & $\mathbf{0 . 1 2 6}$ & $\mathbf{0 . 1 4 6}$ & $\mathbf{0 . 2 4 8}$ & - \\
$A_{\mathrm{Z}}^{4}$ & - & 0.060 & 0.087 & $\mathbf{0 . 1 3 4}$ & - \\
\hline
\end{tabular}

the F10.7 solar flux density. The result supports our previous hypothesis according to which the corresponding EOFs describe the ionospheric current system responsible of the solar quiet $\left(S_{\mathrm{q}}\right)$ daily variation. Indeed, the F10.7 solar parameter is a proxy of the actual solar total irradiance which is well known to affect the status of the upper atmosphere by energizing the current systems of the ionospheric dynamo. Furthermore, it seems that a statistically significant correlation can be found also with the solar wind dynamic pressure $P_{n}^{\mathrm{SW}}$ for the H-component. It can be understood in terms of changes of the magnetospheric field topology (Macmillan and Droujinina, 2007).
Differently, the values of the correlation coefficient $|r|$ for the amplitudes $A_{\mathrm{H}}^{1}, A_{\mathrm{D}}^{2}$ and $A_{\mathrm{Z}}^{2}$ reveal a good degree of correlation with the Dst-index, $B_{\mathrm{Z}}^{\mathrm{SW}}, E_{\mathrm{y}}^{\mathrm{SW}}$ and $P_{n}^{\mathrm{SW}}$ being the found values of correlation greater than three times the $5 \%$ threshold value. This result confirms the hypothesis that the EOFs $\phi_{\mathrm{H}}^{1}(t), \phi_{\mathrm{D}}^{2}(t)$ and $\phi_{\mathrm{Z}}^{2}(t)$, associated with these PCs, mainly describe the current system formed by the partial ring current and the related field-aligned currents. Indeed, it is well known that the intensity of these currents increases with the magnetospheric activity level as well represented by the Dst-index. This point is also confirmed by the high degree of the correlation with the IMF and solar wind parameters (see e.g. $B_{\mathrm{Z}}^{\mathrm{SW}}, E_{\mathrm{y}}^{\mathrm{SW}}$ or $P_{n}^{\mathrm{SW}}$ ), although the correlation degree with $B_{\mathrm{Z}}^{\mathrm{SW}}$ is less than that with $E_{\mathrm{y}}^{\mathrm{SW}}$ and Dst-index. This point can be understood considering that a better proxy of the solar-wind driving is the southward component of $B_{\mathrm{Z}}^{\mathrm{SW}}$, which generally drives the reconnection at the nose of the magnetopause allowing the solar wind to flow into the magnetospheric cavity. Furthermore, the second group of PCs still shows a certain degree of influence from F10.7 solar flux density, suggesting that the NOC decomposition is perhaps not able to completely remove the $S_{\mathrm{q}}$ field from the EOFs associated with this set of PCs. Thus, although the NOC decomposition is substantially an orthogonal decomposition, still a certain cross-talk between the different PCs may exist due to the presence of shared information. This point will be investigated later in this work using an information theory approach.

The third set of PCs, associated with those EOFs that we suppose to describe the magnetopause current system, are mainly correlated with the solar wind parameters along $\mathrm{H}$ and $\mathrm{D}$ components, and Dst-index on $\mathrm{Z}$ component. Again, a certain degree of correlation with the F10.7 solar flux density is still present especially in the D-component.

The fourth set of PCs indicates a good correlation with $B_{\mathrm{Z}}^{\mathrm{SW}}$, the solar wind dynamic pressure $P_{n}^{\mathrm{SW}}$, the electric field 


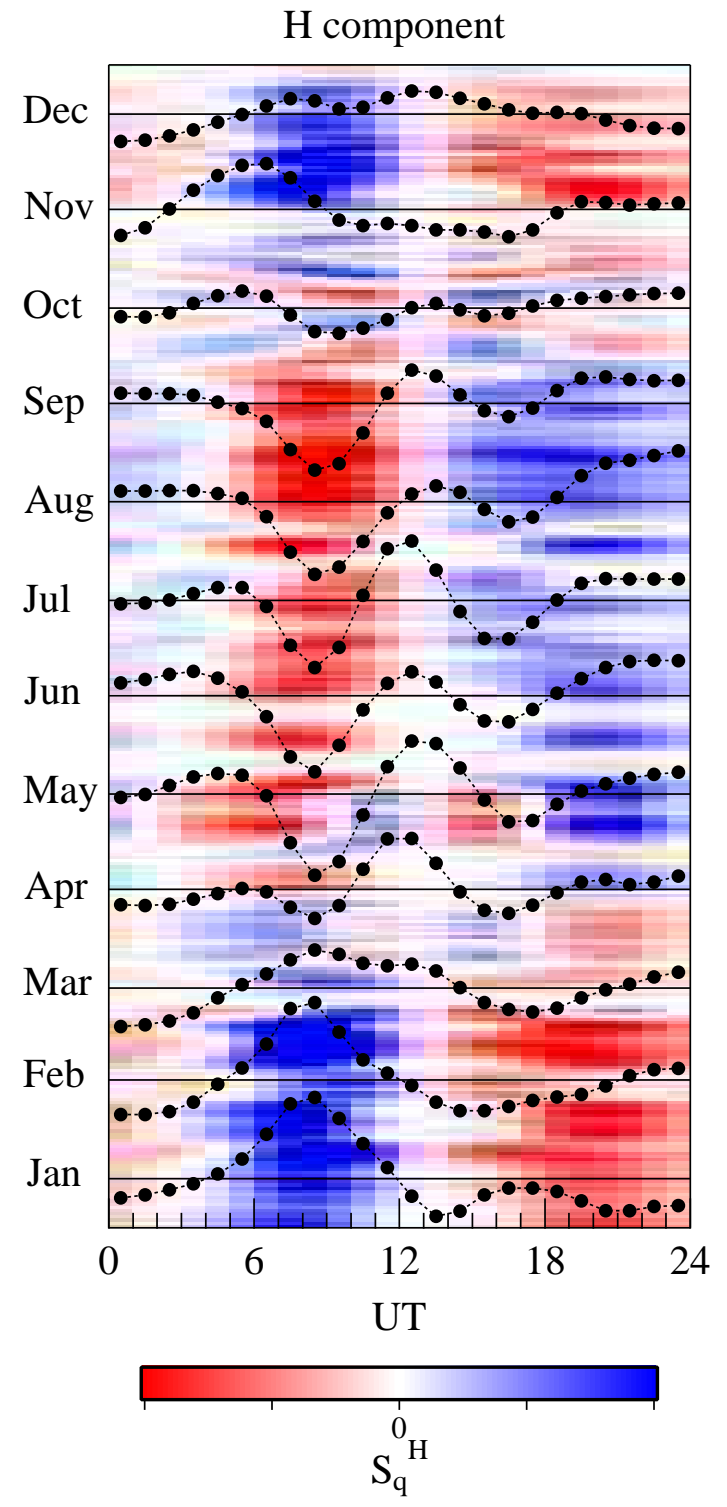

Fig. 12. Comparison between the seasonal dependence of the 1996 quiet daily variation along the $\mathrm{H}$ component as reconstructed using contribution from the 2 nd and 4 th $\operatorname{EOF}\left(S_{\mathrm{q}}^{\mathrm{H}}=A_{\mathrm{H}}^{2} \phi_{\mathrm{H}}^{2}+A_{\mathrm{H}}^{4} \phi_{\mathrm{H}}^{4}\right)$ (negative and positive values are in red and blue, respectively) and the true $S_{\mathrm{q}}$ (black circles) evaluated by averaging the daily variation of the 5 quietest days of every month of 1996. Time is reported in universal time $(\mathrm{UT}=\mathrm{LT}-1)$ for convenience.

$E_{\mathrm{y}}^{\mathrm{SW}}$ and F10.7 supporting the hypothesis that the EOFs associated with these PCs could be representative of mixed current systems affected by changes of interplanetary conditions and that they may include a contribution along the $\mathrm{H}$ and $\mathrm{D}$ elements coming from the ionospheric current system.

In the light of the above correlation studies, we take the opportunity to return to the question of the seasonal phase changes of the quiet daily variation $S_{\mathrm{q}}$. In Sect. 3.1 we proposed that the discrepancies in the phase shift observed com- paring NOC-reconstructed and true $S_{\mathrm{q}}$ could be due to the fact that the effect of the ionospheric current system responsible of the $S_{\mathrm{q}}$ field may influence different EOFs. Here we want to verify this hypothesis. Limiting our discussion to the $\mathrm{H}$ magnetic field component, according to the results reported in Table 1, we may note that the $A_{\mathrm{H}}^{4}$ shows a significant correlation mainly with F10.7. This suggests us that the associated EOF $\left(\phi_{\mathrm{H}}^{4}(t)\right)$ could contain relevant information on the solar quiet daily variation. Figure 12 shows the reconstruction of the solar quiet daily variation $S_{\mathrm{q}}^{\mathrm{H}}$ for the $\mathrm{H}$ magnetic component made taking into account of all the EOFs showing a significant correlation with F10.7, i.e. the 2nd and 4th EOFs. Phase shifts are now visible and a better correlation in phase with true data is observed. This confirms the idea that the effect of a single current may enter into different EOFs although with different weights.

\subsection{PCs cross-talk}

To investigate the actual existence of a certain amount of cross-talk between the different PCs, we have first estimated the orthogonality degree and successively the presence of a nonlinear correlation by means of the mutual information $\operatorname{MI}(X, Y)$.

To evaluate the orthogonality degree between couples of PCs obtained by the NOC decomposition we have defined the following quantity,

$I O_{i j}=\frac{2 \sum_{t} A_{L}^{i}(t) A_{L}^{j}(t)}{\sum_{t}\left(A_{L}^{i}(t)^{2}+A_{L}^{j}(t)^{2}\right)}$

where $A_{L}^{i}(t)$ and $A_{L}^{j}(t)$ are two PCs coming from the NOC decomposition of the same magnetic element $(L=H, D, Z)$. In our case the obtained values of $I O_{i j}$ are in all the cases (with $i \neq j$ ) practically zero $\left(\left|I O_{i j}\right|<10^{-5}\right.$ ), thus confirming the orthogonality of the PCs as expected. As a consequence of this result we can conclude that linear cross-talk is not present among the PCs. However, we cannot exclude that a certain amount of nonlinear cross-talk could exist and that it could be revealed by means of an information theory approach by evaluating the mutual information $\operatorname{MI}(X, Y)$.

The mutual information quantity $\operatorname{MI}(X, Y)$ is indeed capable of better detecting the overall linear and nonlinear coupling between two quantities, in terms of shared information. This quantity is defined as

$\operatorname{MI}(X, Y)=\sum_{x_{i} \in \mathcal{A}} \sum_{y_{j} \in \mathcal{B}} p\left(x_{i}, y_{j}\right) \log \frac{p\left(x_{i}, y_{j}\right)}{p\left(x_{i}\right) p\left(y_{j}\right)}$

where $p\left(x_{i}, y_{j}\right)$ is the joint probability function of finding $\left(x_{i}, y_{j}\right) \in \mathcal{A} \otimes \mathcal{B}$, and $p\left(x_{i}\right)$ and $p\left(y_{j}\right)$ are the probability distribution functions of $X$ and $Y$. Thus, the mutual information can be thought of as a generalized correlation measure, which is sensitive to any relationship between the two signals $X$ and $Y$ (Shannon, 1948; Gelfand et al., 1956). 
The computation of mutual information requires the evaluation of joint and single variable probability distribution functions. This point is so crucial in correctly evaluating the mutual information that different techniques were developed in the past (Kraskov et al., 2004; Cellucci et al., 2005). To overcome this computational problem we apply the standard histogram technique combined with an optimal data-based binning (Knuth et al., 2005; Knuth, 2006). According to Knuth (2006) this optimal data-based binning technique estimates the optimal number of bins in a uniform bin-width histogram deriving the posterior probability for the number of bins in a Bayesian framework. Namely, if $N$ is the total number of samples, $M$ is the number of bins and $\left\{n_{k}\right\}$ in the number of samples in the $k$-th bin, then the posterior probability for the number of bins $p\left(M \mid N, n_{k}\right)$ is

$p\left(M \mid N, n_{k}\right) \propto\left(\frac{M}{V}\right)^{N} \frac{\Gamma(M / 2)}{\Gamma(1 / 2)^{M}} \frac{\prod_{k} \Gamma\left(n_{k}+1 / 2\right)}{\Gamma(N+M / 2)}$,

where $V$ is the data range. The optimal number of bins is given by the maximum value of the probability $p\left(M \mid N, n_{k}\right)$. Equation (9) can be extended to higher dimensions to evaluate n-dimensional distribution functions (see Knuth, 2006, for more details).

In Table 2 we report the mutual information values $\operatorname{MI}\left(A_{L}^{i}, A_{L}^{j}\right)$ between the couples of PCs $A_{L}^{k}$ of the same magnetic field component. We limit our analysis only to the first 4 PCs.

To estimate the statistical significance of $\operatorname{MI}\left(A_{L}^{i}, A_{L}^{j}\right)$ values we evaluate a threshold value for $\operatorname{MI}\left(A_{L}^{i}, A_{L}^{j}\right)$, which corresponds to the usual $5 \%$ null-hypothesis value by applying the same technique described in the previous section for the Pearson's correlation coefficient based on SDT. We report in Table 2 the corresponding 5\% significance threshold value for each couple of PCs. As in the previous case, values of $\operatorname{MI}\left(A_{L}^{i}, A_{L}^{j}\right)$ less than the corresponding $5 \%$ significance threshold value are read as absence of nonlinear cross-talk.

Looking at the results reported in Table 2, we can confirm our previous interpretation on the existence of a certain nonlinear cross-talk among the different PCs, confirming the hypothesis according to which the EOFs and PCs from NOC decomposition may still be, in some cases, representative of mixed current systems.

\subsection{Statistical and spectral features of PCs}

To complete our analysis of PCs we investigate their statistical and spectral features.

Figure 13 reports the power spectral density (PSD) of the four sets of PCs, grouped as in Table 1. These PSDs are evaluated as the trace of the spectral density matrix, i.e., $S(f)=\sum_{i} S_{i}(f)$ where the $S_{i}(f)$ are the PSDs of the $\mathrm{H}$ and Z PCs of each group (here D-component has not been considered being representative of an angular variation). All the PSDs exhibit the characteristic annual variation along
Table 2. The mutual information $\operatorname{MI}\left(A_{L}^{i}, A_{L}^{j}\right)$ between the PCs of the same NOC decomposition $(L=H, D$ or $Z$ ). Values in round brackets are the corresponding $5 \%$ significance threshold values. The lowercase $L$ stands for $H, D$ and $Z$ magnetic field elements, respectively. Remind that $\operatorname{MI}\left(A_{L}^{i}, A_{L}^{j}\right)=M I\left(A_{L}^{j}, A_{L}^{i}\right)$, being mutual information a symmetric quantity.

\begin{tabular}{|c|c|c|c|}
\hline & $A_{L}^{2}$ & $A_{L}^{3}$ & $A_{L}^{4}$ \\
\hline \multicolumn{4}{|c|}{ H Component } \\
\hline$A_{L}^{1}$ & $\begin{array}{c}0.050 \\
(0.016)\end{array}$ & $\begin{array}{c}0.092 \\
(0.016)\end{array}$ & $\begin{array}{c}0.058 \\
(0.014)\end{array}$ \\
\hline$A_{L}^{2}$ & & $\begin{array}{c}0.067 \\
(0.040)\end{array}$ & $\begin{array}{c}0.041 \\
(0.018)\end{array}$ \\
\hline$A_{L}^{3}$ & & & $\begin{array}{c}0.036 \\
(0.017)\end{array}$ \\
\hline \multicolumn{4}{|c|}{ D Component } \\
\hline$A_{L}^{1}$ & $\begin{array}{c}0.227 \\
(0.134)\end{array}$ & $\begin{array}{c}0.075 \\
(0.049)\end{array}$ & $\begin{array}{c}0.042 \\
(0.018)\end{array}$ \\
\hline$A_{L}^{2}$ & & $\begin{array}{c}0.046 \\
(0.032)\end{array}$ & $\begin{array}{c}0.028 \\
(0.015)\end{array}$ \\
\hline$A_{L}^{3}$ & & & $\begin{array}{c}0.061 \\
(0.015)\end{array}$ \\
\hline \multicolumn{4}{|c|}{ Z Component } \\
\hline$A_{L}^{1}$ & $\begin{array}{c}0.082 \\
(0.022)\end{array}$ & $\begin{array}{c}0.047 \\
(0.037)\end{array}$ & $\begin{array}{c}0.034 \\
(0.019)\end{array}$ \\
\hline$A_{L}^{2}$ & & $\begin{array}{c}0.043 \\
(0.016)\end{array}$ & $\begin{array}{c}0.039 \\
(0.015)\end{array}$ \\
\hline$A_{L}^{3}$ & & & $\begin{array}{c}- \\
(0.017)\end{array}$ \\
\hline
\end{tabular}

with its higher harmonics. An extra characteristic periodicity at $f \sim 0.037 \mathrm{day}^{-1}$ is found in the case of the SD daily variation (plot \#2 of Fig. 13). It corresponds to the well-known Carrington periodicity relative to solar rotation ( $\sim 27.28$ day). The obtained result supports the identification of $\phi_{\mathrm{H}}^{1}(t), \phi_{\mathrm{D}}^{2}(t)$ and $\phi_{\mathrm{Z}}^{2}(t)$ as representative of the disturbance daily variation suggesting the clear influence of the recurrent solar structures on the variations of the partial ring current and field aligned current features. We also notice that the PSD reported in plot \#4 of Fig. 13 exhibits, besides the annual periodicity and its harmonic and/or sub-harmonic, a small peak at the second harmonic of the Carrington solar rotation periodicity. That confirms the previous hypothesis of a combination of current systems, which are described by EOFs $\phi_{\mathrm{H}}^{4}(t), \phi_{\mathrm{D}}^{4}(t)$ and $\phi_{\mathrm{Z}}^{4}(t)$, and that we are not able to identify correctly.

At the end, Fig. 14 shows the probability distribution functions (PDFs) of the first four PC groups $A_{L}^{k}(k=1,2,3,4)$ for the three magnetic field elements $(L=H, D, Z)$, clustered according to the previous classification (see Sect. 3.1). To compare the different statistics of the PCs for the different 

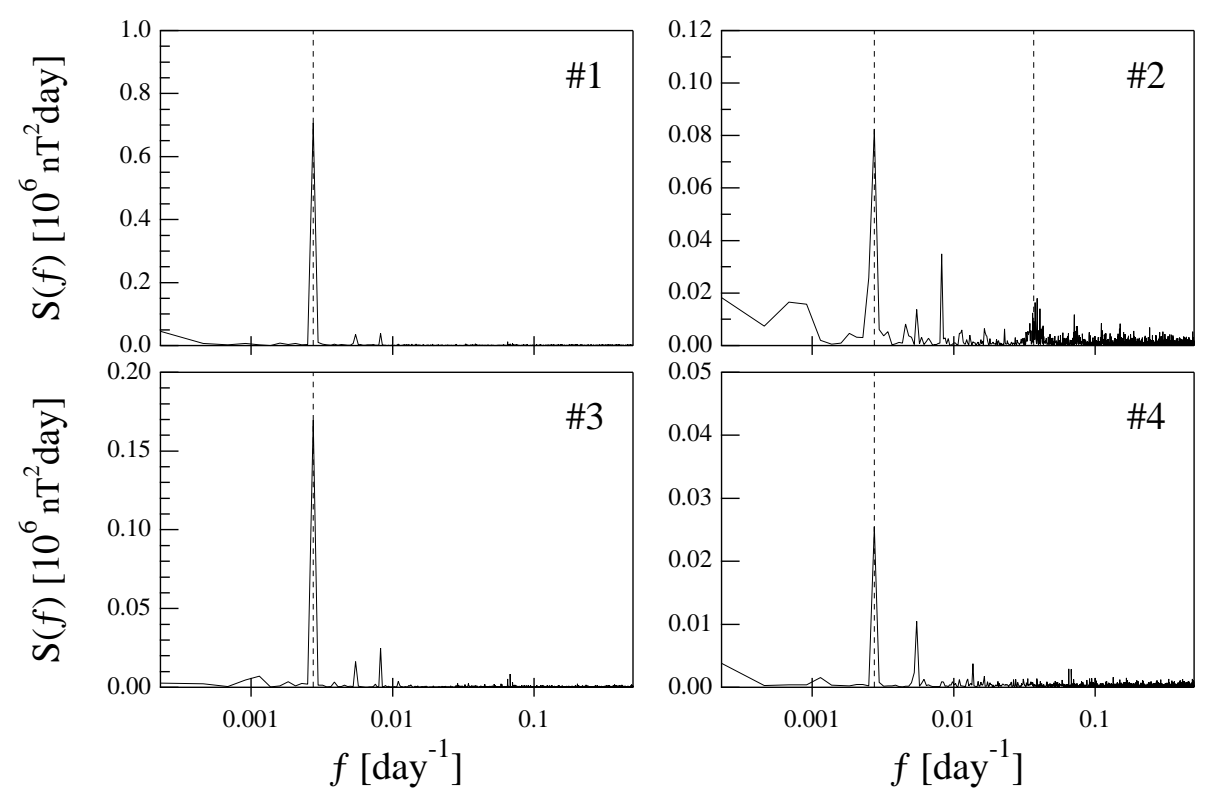

Fig. 13. The trace of the spectral density matrix $S(f)$ for the first four PCs. The vertical dashed lines refer to the annual periodicity $f \sim .0027$ day $^{-1}$ and to the characteristic mean solar rotation periodicity $f \sim 0.037$ day $^{-1}$ (Carrington's periodicity).
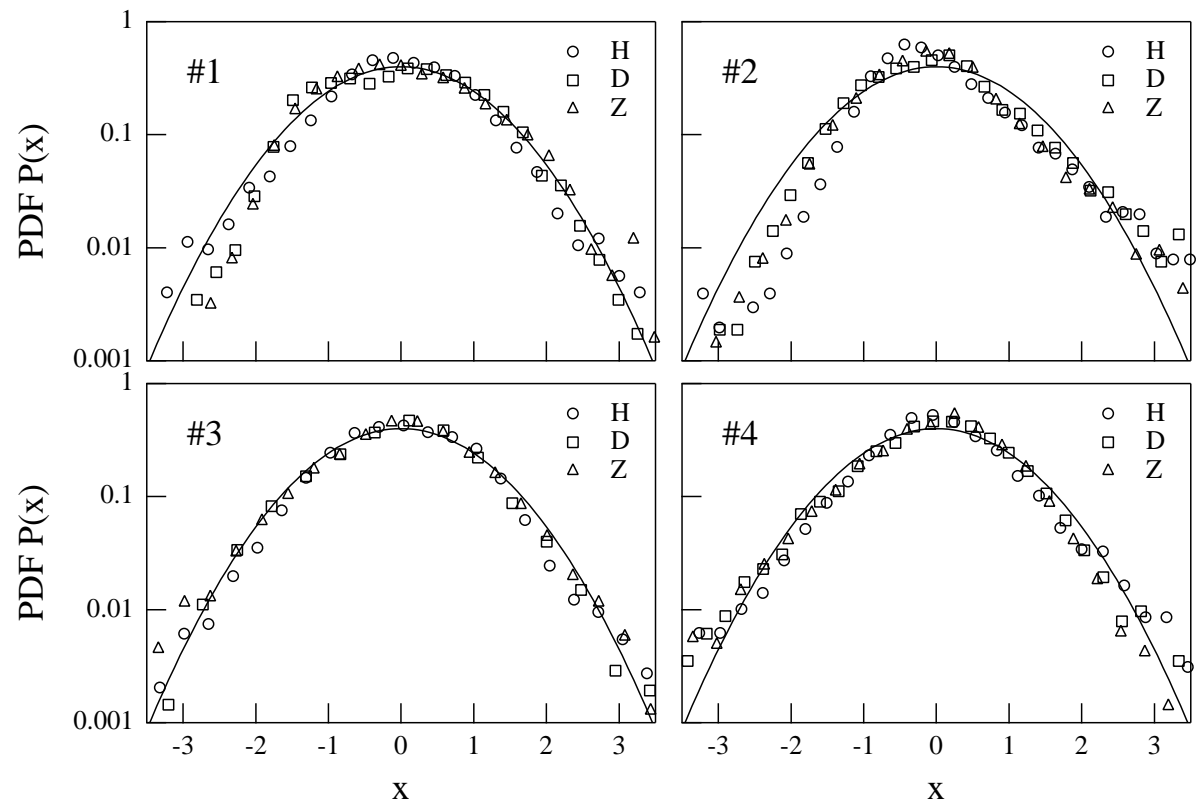

Fig. 14. The probability distribution functions of the first four PCs for the three magnetic field elements. Plot \#1 and \#2 refer to $S_{\mathrm{q}}$ and $S_{\mathrm{D}}$ daily variations as identified in the previous section. The solid line refers to a unit-variance zero-mean Gaussian distribution.

elements before computing the PDFs we shifted each PC by its mean value and normalized to the variance: $A_{L}^{k} \rightarrow x=$ $\left(A_{L}^{k}-\left\langle A_{L}^{k}\right\rangle\right) / \sigma_{A_{L}^{k}}$. We note that the statistics of the PCs associated with the $S_{\mathrm{q}}$ daily variation (plot \#1 of Fig. 14) is in agreement with a Gaussian distribution, while in the case of the other PCs the Gaussian character is less evident or totally lost. It is particularly true in the case of the PDFs of the PCs associated with the $S_{\mathrm{D}}$ daily variation (plot \#2 of Fig. 14), which exhibits a pronounced skewness resulting similar to the PDF of Dst-index values.

\section{Conclusions}

The study of magnetic perturbations and their interpretation as current systems flowing in the Earth and in space is 
extremely complicated. The daily ground magnetic perturbations are a superposition of contributions from the horizontal ionospheric currents, field-aligned currents, currents in the magnetosphere, and currents induced at the Earth's surface. Recently, Xu and Kamide (2004) and Chen et al. (2007) have shown that it is possible to separate and recognize the different current systems that contribute to the geomagnetic field daily variation applying the Natural Orthogonal Components technique. In this work, we apply this method to a geomagnetic dataset recorded at L'Aquila geomagnetic observatory during a time interval of 12 years. Namely, we study the temporal evolution, the spectral and statistical properties of the amplitudes associated with the different EOFs, and investigate the correlations between these amplitudes and a set of descriptors of the magnetospheric and ionospheric dynamics during one solar cycle (from 1993 to 2004). The found results allowed us to reconstruct the 3-dimensional structure of the different ionospheric and magnetospheric current systems which contribute to the geomagnetic daily variation.

According to our analysis, the three principal contributions to the geomagnetic daily variations are related to: (i) the ionospheric currents, (ii) the current system in the ionosphere and magnetosphere corresponding to the partial ring current and the related field-aligned currents, and (iii) the magnetopause currents. However, we note that NOC analysis on the three magnetic field elements may not be able to completely separate the various contributions. The found EOFs may include the contributions from two or more current systems or, conversely, the effect of a single current may enter into different EOFs. This might be due to the fact that the magnetic fields generated by different current systems in the ionosphere and magnetosphere may interfere nonlinearly. In this case, as already mentioned, NOC technique is not able to completely separate the different contributions.

Furthermore, the influence of these current systems on $\mathrm{H}$, $\mathrm{D}$ and $\mathrm{Z}$ magnetic field elements is similar but not equal. The horizontal component of the geomagnetic field $(\mathrm{H})$ is characterized by an eigenvalue spectrum where the first two terms are of the same order in energy. In this case, both the ionospheric wind dynamo currents and the current system formed by the partial ring current and the field-aligned currents contribute with an equal weight to the reconstruction of the geomagnetic daily variation. In contrast, the other two elements (D and Z) of the geomagnetic field exhibit an eigenvalue spectrum where the first eigenvalue is about one order greater than the others and, therefore, the ionospheric currents carry out a fundamental role in the structure of the geomagnetic daily variation in respect to the other magnetospheric current systems.

To support our interpretation of EOFs in terms of the various ionospheric and magnetospheric current systems, we study the correlation between the associated amplitudes (PCs) and a set of parameters, generally used as descriptors of the magnetospheric and ionospheric dynamics and solar wind changes. The results obtained indicate a good "corre- lation", being the found values of correlation much greater than the 5\% threshold value, between the PCs and those parameters that are responsible for the evolution of the current systems associated with the corresponding EOFs, showing how in some cases there could be a certain amount of crosstalk among the different sets of EOFs. This point has been substantiated by the investigation of nonlinear coupling using the mutual information. Moreover, in some cases there is also a coincidence between the probability distribution functions of the PCs and the external parameters that describe the temporal evolution of these currents. The study of the probability distribution functions of all the examined PCs suggests that temporal fluctuations of the different current systems, contributing to the geomagnetic daily variation, are peculiar of systems that develop through a non-equilibrium dynamics near a stationary state where sporadic large fluctuations may occur with a probability higher than for equilibrium Gaussian fluctuations (Consolini et al., 2008).

Acknowledgements. We acknowledge the NSSDC/OMNI database PIs (J. H. King and N. Papitashvili, ADNET, NASA GSFC) and CDAWeb for the interplanetary and magnetospheric index data used in this work and available on web.

The authors would like to thank also L'Aquila magnetic observatory for supplying the data, which are used in the study.

Topical Editor K. Kauristie thanks two anonymous referees for their help in evaluating this paper.

\section{References}

Campbell, W. H.: Geomagnetic storms, the Dst ring current myth and lognormal distributions, J. Atmos. Terr. Phys., 58, 11711187, 1996.

Campbell, W. H.: Introduction to geomagnetic fields, 2nd Edition, Cambridge University Press, 2003.

Cellucci, C. J., Albano, A. M., and Rapp, P. E.: Statistical validation of mutual information calculations: Comparison of alternative numerical algorithms, Phys. Rev. E, 71, 066208 , doi:10.1103/PhysRevE.71.066208, 2005.

Chapman, S.: On the theory of the solar diurnal variation of the Earth's magnetism, Proc. R. Soc. Lond. Ser. A, 129, 369-386, 1929.

Chapman, S. and Bartels, J.: Geomagnetism, Oxford University Press, New York 1940.

Chen, G. X., Xu, W. Y., Du, A. M., Wu, Y. Y., Chen, B., and Liu, X. C.: Statistical characteristics of the day-to-day variability in the geomagnetic $S q$ field, J. Geophys. Res. E, 112, A06320, doi:10.1029/2006JA012059, 2007.

Chulliat, A., Blanter, E., Le Mouel, J. L., and Shnirman, M.: On the seasonal asymmetry of the diurnal and semidiurnal geomagnetic variations, J. Geophys. Res., 110, A05301, doi:10.1029/2004JA010551, 2005.

Consolini, G., De Michelis, P., and Tozzi, R.: On the Earth's magnetospheric dynamics: Nonequilibrium evolution and the fluctuation theorem, J. Geophys. Res., 113, A08222, doi:10.1029/2008JA013074, 2008.

Consolini, G., Tozzi, R., and De Michelis, P.: Complexity in the sunspot cycle, Astron. Astrophys., 506, 1381-1391, 2009. 
Gelfand, I. M., Kolmogorov, A. N., and Yaglom, A. M.: On the general definition of an amount of information, Dokl. Akad. Nauk SSSR, 111, 745-748, 1956.

Golovkov, V. P., Papitashvili, N. E., Tyupkin, Y. S., and Kharin, E. P.: Division of variations of geomagnetic field on a quiet and disturbed components by a method of natural orthogonal components, Geomagn. Aeron., 18, 511-515, 1978.

Golovkov, V. P., Papitashvili, V. O., and Papitashvili, N. E.: Automated calculation of the $\mathrm{k}$ indices using the method of natural orthogonal components, Geomagn. Aeron., 29, 667-670, 1989.

Golovkov, V. P., Kozhoeva, K. G., and Shkolnikova, S. I.: Application of the method of natural orthogonal components for separation of partially nonorthogonal variations, Geomagn. Aeron., 32, 162-165, 1992.

Howe, H. H.: An anomaly of the geomagnetic daily variation at Honolulu, J. Geophys. Res., 55, 271-274, 1950.

Jackson, G. M., Mason, I. M., and Greenhalgh, S. A.: Principal component transforms of triaxial recordings by singular value decomposition, Geomagn. Aeron., 56, 528-533, 1991.

Knuth, K. H.: Optimal data-based binning for histograms, ArXiv: physics/0605197v1, 2006.

Knuth, K. H., Gotera, A., Curry, C. T., Huyser, K. A., Wheeler, K. R., and Rossow, W. B.: Revealing relationships among relevant climate variables with information theory, in: Proc. of the Earth-Sun System Technology Conference (ESTC 2005), Adelphi (MD), 2005.

Kraskov, A., Stögbauer, H., and Grassberger, P.: Estimating mutual information, Phys. Rev. E, 69, 066138, doi:10.1103/PhysRevE.69.066138, 2004.

Macmillan, S. and Droujinina, A.: Long-term trends in geomagnetic daily variation, Earth Planets Space, 59, 391-395, 2007.

Matsushita, S. and Maeda, H.: On the geomagnetic quiet daily variation field during the IGY, J. Geophys. Res., 70, 2535-2558, 1965.

Merrill, R. T., McElhinny, M. W., and McFadden, P. L.: The Magnetic Field of the Earth, Paleomagnetism, the Core and the Deep Mantle, Academic Press, San Diego, CA, 1996.

Mininni, P. D., Gomez, D. O., and Mindlin, G. B.: Biorthogonal Decomposition Technique unveil the nature of the irregularities observed in the solar cycle, Phys. Rev. Lett., 89, 061101, doi:10.1103/PhysRevLett.89.061101, 2002.
Mininni, P. D., Lopez Fuentes, M., and Mandrini, C. H.: Study of bi-orthogonal modes in magnetic butterflies, Solar Phys., 219, 367-378, 2004.

Papitashvili, N. E., Papitashvili, V. O., Belov, B. A., Hakkinen, L., and Sucksdorff, C.: Magnetospheric contribution to k-indexes, Geophys. J. Int., 111, 348-356, 1992.

Richmond, A. D., Matsushita, S., and Tarpley, J. D.: On the production mechanism of electric currents and fields in the ionosphere, J. Geophys. Res., 81, 547-555, 1976.

Rotanova, N. M., Papitashvili, N. Y., and Pushkov, A. N.: Spacetime analysis of 60-year variations of the field by the data of the world network of observatories, Geomagn. Aeron., 22, 847-851, 1982.

Shannon, C. E.: A mathematical theory of communication, Bell Systems Technol. J., 27, 397-423 (part I), 623-656 (part II), 1948.

Sugiura, M. and Kamei, T.: Equatorial Dstindex 1957-1986, in IAGA Bull., 40, ISGI Publications Office, Saint-Maur-desFosses, France, 1991.

Sun, W., Xu, W. Y., and Akasofu, S. I.: Mathematical separation of directly driven and unloading components in the ionospheric equivalent currents during substorms, J. Geophys. Res., 103, 11695-11700, 1998.

Sun, W., Xu, W. Y., and Akasofu, S. I.: An improved method to deduce the unloading component for the magnetospheric substorms, J. Geophys. Res., 105, 13131-13140, 2000.

Theiler, J., Eubank, S., Longtin, A., Galdrikian, B., and Farmer, J. D.: Testing for nonlinearity in time series: the method of surrogate data, Physica D, 58, 77-94, 1992.

$\mathrm{Xu}$, W. Y.: Revision of the high-degree Gauss coefficients in the IGRF 1945-1955 models by using natural orthogonal component analysis, Earth, Planets and Space, 54, 753-761, 2002.

$\mathrm{Xu}$, W. Y.: NOC model of the Earth's main magnetic field, Sci. China D, 46, 882-894, 2003.

$\mathrm{Xu}, \mathrm{W}$. Y. and Kamide, Y.: Decomposition of daily geomagnetic variations by using method of natural orthogonal component, J. Geophys. Res., 109, A05218, doi:1029/2003JA010216, 2004. 